\title{
La Reforma Financiera y uso del crédito en el desarrollo de las empresas en México
}

The Financial Reform and Use of Credit in the Enterprises in Mexico

\author{
Genaro Sánchez Barajas* :
}

\begin{abstract}
Resumen
A un año y medio de haber entrado en vigor la reforma financiera, ésta falló en la promoción vía el crédito bancario aplicado al desarrollo empresarial, bujía del desarrollo económico nacional, en virtud de que disminuyó el número total de empresas de 2009 a 2014, crecieron la micro y grandes empresas pero en las primeras disminuyó su producción y las segundas, desde hace tiempo monopolizan la producción de nuestra economía; todo ello debido en mucho a que la SHCP para la elaboración de la política pública correspondiente anualmente, no analiza los resultados de operación de las empresas como tampoco tiene en cuenta la opinión que periódicamente suelen dar los empresarios a INEGI.
\end{abstract}

\section{Palabras clave:}

- Politica fiscal

- Medición del crecimiento económico

- Análisis de la producción y localización de empresas

JEL: E62, O47, R3

\begin{abstract}
A year and a half after entry into force of financial reform, it failed in the promotion via bank credit applied to business development, spark plug of national economic development, under which decreased the total number of enterprises in 2009-2014, they grew micro and large companies but declined in the first and second production has long monopolized the production of our economy; all due greatly to the SHCP for the preparation of the corresponding annual public policy, it does not discuss the results of operations of the companies and does not take into account the views that often give the employer periodically to INEGI.
\end{abstract}

\section{Keywords:}

- Fiscal Policy

- Measurement of Economic Growth

- Production Analysis and Firm Location

\section{Introducción}

El 9 de enero de 2014 (Internet, 2015) la Secretaría de Hacienda y Crédito Público dio a conocer en el Diario Oficial de la Federación (DOF) el decreto por el que se reforman, adicionan y derogan diversas disposiciones en materia financiera y se expide la Ley para Regular las Agrupaciones Financieras. Dicha normatividad es el sustento de la reforma financiera que a un año y medio de haber sido instrumentada conviene reflexionar sobre si el crédito, eje rector de muchas de sus acciones, efectivamente tiene la importancia que le atribuyó el Ejecutivo Federal cuando la dio a conocer como una de las principales reformas estructurales que necesitaba México para mejorar la calidad de vida de sus ciudadanos. En otras palabras, dada la gran promoción que institucionalmente se le ha hecho al crédito para que los empresarios lo usen como palanca del desarrollo competitivo de sus empresas,

* Es PH. D. por la Russian Academy of Sciences, profesor en la licenciatura y en la maestría en economía y tutor en los doctorados de Economía y de Ciencias de la Administración de la UNAM. Domicilio: Cubículo 15, Edificio A de la Facultad de Economía, Cd. Universitaria, D.F; correo: genarosa@unam.mx. 
conviene preguntarse si con base en la opinión de los empresarios y del análisis de la operación de sus unidades productivas y de servicios, ¿La escasez del crédito es realmente uno de sus principales problemas que les impiden ser competitivos y por consiguiente, gestar el círculo virtuoso de aprovechar mejor la capacidad de su planta productiva en la creación de los mínimos de bienestar que requiere la sociedad, con empleos permanentes, bien remunerados y sustentablemente?

Contestar esta pregunta es el objetivo de este estudio. Así, como referencia es necesario delinear el contenido de la reforma financiera que tiene como antecedente el marco del Pacto por México; en él se indica que es menester ampliar y mejorar el crédito; para ello en la reforma financiera se modificaron 34 ordenamientos contenidos en 13 iniciativas y su consecución consta de cuatro pilares: fomentar la competencia del sistema financiero y el crédito a través de la banca de desarrollo, ampliar el crédito a través de instituciones privadas y dar solidez y prudencia al sistema financiero en su conjunto, (ídem).

El primer pilar se refiere al fomento de la competencia en el sector financiero y al fortalecimiento de las atribuciones de la Comisión Nacional para la Protección y Defensa de Usuarios de los Servicios Financieros (Condusef) para incluir mayores obligaciones de transparencia en las instituciones financieras.

Con el segundo pilar se busca fomentar el crédito a través de la banca de desarrollo como un motor del crecimiento económico, sobre todo en áreas de infraestructura, apoyo a las pequeñas y medianas empresas, así como la innovación y la creación de patentes. Como se informa, en este punto, el mandato específico es promover la perspectiva de género, por lo que la banca de desarrollo fomentará una mayor inclusión financiera de las mujeres.

El tercer pilar de la reforma financiera consiste en ampliar el crédito a través de las instituciones financieras privadas y que los depósitos de los ahorradores sean canalizados al crédito productivo, además de simplificar los regímenes para el otorgamiento y ejecución de garantías crediticias y lograr una reducción de los riesgos y con ello de los costos del crédito.

Como cuarto pilar de las modificaciones en materia financiera está dar mayor solidez y prudencia al sector financiero en su conjunto. En este contexto, en el documento emitido en el DOF se establecen los aspectos que buscan el fortalecimiento de la Condusef, que tendrá a su cargo la organización, funcionamiento y promoción del Sistema Arbitral en Materia Financiera, ibid.

En este contexto, por la importancia económica de esta reforma es que decidí analizar sus fundamentos para después verificar si efectivamente se está en 
el camino correcto de lograr un desarrollo económico sostenido y sustentable, usando el crédito como uno de los principales instrumentos institucionales que demandan los empresarios para coadyuvar en el crecimiento y desarrollo económico del país. Para ello tomaré como guía los datos sobre la operación de las empresas y la información que los empresarios le proporcionan al gobierno de la República porque, supongo, son algunas de las fuentes principales que toma en cuenta para elaborar anualmente la política pública de fomento que necesitan para dinamizar la economía nacional. La información disponible hasta la fecha está contenida en:

1.- Los Censos Económicos de 1999, 2009 y los datos definitivos y preliminares ( muy generales) del Censo Económico de 2014

2.- La Encuesta Nacional de Micronegocios, Enamin, de 2013, porque intuyo que fue una de las referencias inmediatas cuyos datos supongo que sirvieron al Ejecutivo Federal para la elaboración de la iniciativa de reforma financiera que presentó al H. Congreso de la Unión, para su aprobación corespondiente.

\section{Hipótesis de trabajo}

1. Para operar las empresas usan el crédito para capital de trabajo;

2. Para operar las empresas usan el crédito para la ampliación o reposición de activos fijos;

3. Para operar las empresas usan el crédito para sustituir procesos de fabricación que sean contaminantes u obsoletos.

Verificación: Empresas manufactureras, evolución de su operación descrita con indicadores que producen resultados en el corto y mediano plazo

Decidí estudiar los resultados de operación de estas empresas de 1999 a 2009 descritos en los Censos Económicos de esos años y de 2014, por tamaño de empresa, por ser ellas las que históricamente han sido identificadas como las apropiadas para dinamizar la economía con el valor agregado que producen y con el empleo permanente y bien remunerado que generan: 29.1 y $23.5 \%$ del total, respectivamente en 2013 según INEGI, Censos Económicos 2014. Para ello utilizaré indicadores que producen efectos en el corto y mediano plazo como son el número de empresas, el personal ocupado, sus remuneraciones, el valor agregado censal bruto y los activos fijos con los que producen sus bienes terminados. 
Análisis de su operación en 1999, por tamaño de empresa. Ese año se registraron 344,118 empresas en el sector, de ellas $90.1 \%$ eran micro, $6.6 \%$ pequeña, $2.4 \%$ medianas y $0.9 \%$ restante eran grandes, como lo ilustra el cuadro 2. Estas Unidades de producción dieron ocupación a 4232322 personas, de las cuales $18.3 \%$ lo hizo en las micro, $11.8 \%$ en las pequeñas, $21.7 \%$ en las medianas y $48.2 \%$ informó que laboraba en las grandes escalas de producción manufacturera. Estos trabajadores percibieron en esa ocasión 199,044 millones de pesos, $3.6 \%$ de esa cantidad en la micro, $7.5 \%$ en la pequeñas, $22.1 \%$ en las medianas y $66.8 \%$ en las grandes empresas. Su complemento como factor de la producción, los activos fijos de las empresas, en 1999 ascendieron a 887 556 millones de pesos; $10.0 \%$ de esa inversión en planta se hizo en micro empresas, $4.5 \%$ en pequeñas, $19.4 \%$ en medianas y $66.1 \%$ en las grandes. La combinación de los factores de la producción: trabajo y capital, en 1999 dio lugar a un valor agregado censal bruto de 581113 millones de pesos. El 8.0\% lo generó la micro, $6.1 \%$ la pequeña, $20.1 \%$ la mediana y $65.8 \%$ restante, la gran industria.

\section{Cuadro I}

Criterios de estratificación empresarial: número de trabajadores

\begin{tabular}{|l|c|c|c|}
\hline \multicolumn{1}{|c|}{ Tamaño de empresa } & Industria & Comercio & Servicios \\
\hline Micro & hasta 10 & hasta 10 & hasta 10 \\
\hline Pequeña & 11 a 50 & 11 a 30 & 11 a 50 \\
\hline Mediana & 51 a 250 & 31 a 100 & 51 a 100 \\
\hline Grande & 251 y más & 101 y más & 101 y más \\
\hline
\end{tabular}

Fuente: elaboración propia a partir de los Censos Económicos 2003: micro pequeñas medianas y grandes empresas.

Comentarios: Como se observa en el cuadro 2, la gran empresa manufacturera con apenas $0.88 \%$ de las unidades de producción, destacó significativamente ya que con $66.10 \%$ del total de la inversión en activos fijos, ocupó a $48.27 \%$ de las personas, que percibieron $66.76 \%$ de las remuneraciones, mismas que al operar la planta productiva generaron $65.84 \%$ del valor agregado censal bruto manufacturero en 1999. Esta prevalencia induce a pensar que no tienen problemas de financiamiento (Sánchez: 2007). 


\section{Cuadro 2}

Valores absolutos y estructura porcentual de los principales indicadores por tamaño de empresa en 1999

\begin{tabular}{|c|c|c|c|c|c|c|c|c|c|c|}
\hline \multirow{2}{*}{$\begin{array}{l}\text { Subsector y } \\
\text { tamaño }\end{array}$} & \multicolumn{2}{|c|}{$\begin{array}{l}\text { Unidades } \\
\text { económicas }\end{array}$} & \multicolumn{2}{|c|}{$\begin{array}{c}\text { Personal ocupado } \\
\text { total }\end{array}$} & \multicolumn{2}{|c|}{ Remuneraciones } & \multicolumn{2}{|c|}{$\begin{array}{l}\text { Valor agregado } \\
\text { censal bruto }\end{array}$} & \multicolumn{2}{|c|}{$\begin{array}{c}\text { Total de } \\
\text { activos fijos }\end{array}$} \\
\hline & Absoluto & $\%$ & Absoluto & $\%$ & $\begin{array}{l}\text { Millones } \\
\text { de pesos }\end{array}$ & $\%$ & $\begin{array}{l}\text { Millones } \\
\text { de pesos }\end{array}$ & $\%$ & $\begin{array}{l}\text { Millones } \\
\text { de pesos }\end{array}$ & $\%$ \\
\hline $\begin{array}{l}\text { Total } \\
\text { Manufacturas }\end{array}$ & 344118 & 100 & 4232322 & 100 & 199044 & 100 & 581113 & 100 & 887556 & 100 \\
\hline Micro & 310118 & 90.12 & 773288 & 18.27 & 7341 & 3.69 & 46172 & 7.95 & 89139 & 10.04 \\
\hline Pequeña & 22739 & 6.61 & 499473 & 11.80 & 14907 & 7.49 & 35328 & 6.08 & 40055 & 4.51 \\
\hline Mediana & 8228 & 2.39 & 916654 & 21.66 & 43910 & 22.06 & 117020 & 20.14 & 172663 & 19.45 \\
\hline Grande & 3033 & 0.88 & 2042907 & 48.27 & 132886 & 66.76 & 382593 & 65.84 & 586698 & 66.10 \\
\hline
\end{tabular}

Fuente: elaboración propia con base en los Censos Económicos de 1999. Los datos de 1999 los clasifiqué tomando como referencia los criterios de estratificación empresarial vigentes en 2004 y 2009.

El sector manufacturero nacional en 2009. Se entrevistaron 436851 empresas en el sector, de ellas $92.52 \%$ eran micro; $5.12 \%$, pequeñas; $1.63 \%$ medianas y $0.74 \%$ restante eran grandes, como lo ilustra el cuadro 3. Estas Unidades de producción dieron ocupación a 4661062 personas, de las cuales $23.19 \%$ lo hizo en las micro, $10.02 \%$ en las pequeñas, $17.12 \%$ en las medianas y $49.67 \%$ fue detectado laborando en las grandes empresas manufactureras. Estos trabajadores percibieron ese año 367,793 millones de pesos, $4.30 \%$ de esa cantidad en la micro, $6.58 \%$ en la pequeñas, $17.78 \%$ en las medianas y $71.34 \%$ en las grandes empresas. Su complemento como factor de la producción, los activos fijos de las empresas, en 2009 ascendieron a 1586991 millones de pesos; 4.09\% de esa inversión en planta se hizo en micro empresas, $4.40 \%$ en pequeñas, $17.64 \%$ en medianas y $73.87 \%$ en las grandes. La combinación de los factores de la producción trabajo y capital, en 2009 dio lugar a un valor agregado censal bruto de 1480821 millones de pesos. El 2.85\% lo generó la micro, $4.29 \%$ la pequeña, $17.51 \%$ la mediana y $75.35 \%$ restante, la gran industria.

Comentarios: en 2009 como en 1999, la gran empresa manufacturera con apenas $0.7 \%$ de las unidad de producción, destacó significativamente ya que con $73.9 \%$ del total de la inversión en activos fijos, dio empleo a $49.7 \%$ de las personas, que allí percibieron $71.3 \%$ de las remuneraciones, mismas que al operar la planta productiva generaron $75.3 \%$ del valor agregado censal bruto manufacturero en 2009. Así, también se intuye que no tienen problemas de crédito. 


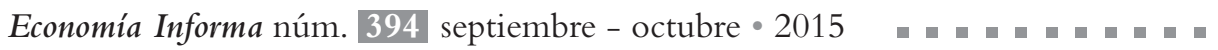

Puede decirse que en estos diez años de análisis la gran empresa aumentó su participación en detrimento de los otros tres estratos empresariales, cuyos indicadores importantes muestran un retroceso, como se muestra a partir del cuadro 3. Si atendemos a las definiciones que hacen de competitividad el Instituto Mexicano de Competitividad: IMCO, y el Foro Económico Mundial de la competitividad, WEF, de que son generadoras de valor agregado, se intuye que las empresas grandes son competitivas (Sánchez: 2012).

Cuadro 3

Valores absolutos y estructura porcentual de los principales indicadores por tamaño de empresa en 2009

\begin{tabular}{|c|c|c|c|c|c|c|c|c|c|c|}
\hline \multirow{2}{*}{$\begin{array}{l}\text { Subsector y } \\
\text { tamaño }\end{array}$} & \multicolumn{2}{|c|}{$\begin{array}{c}\text { Unidades } \\
\text { económicas }\end{array}$} & \multicolumn{2}{|c|}{$\begin{array}{c}\text { Personal ocupado } \\
\text { total }\end{array}$} & \multicolumn{2}{|c|}{ Remuneraciones } & \multicolumn{2}{|c|}{$\begin{array}{c}\text { Valor agregado } \\
\text { censal bruto } \\
\end{array}$} & \multicolumn{2}{|c|}{$\begin{array}{c}\text { Total de } \\
\text { activos fijos }\end{array}$} \\
\hline & Absoluto & $\%$ & Absoluto & $\%$ & $\begin{array}{l}\text { Millones } \\
\text { de pesos }\end{array}$ & $\%$ & $\begin{array}{l}\text { Millones } \\
\text { de pesos }\end{array}$ & $\%$ & $\begin{array}{l}\text { Millones } \\
\text { de pesos }\end{array}$ & $\%$ \\
\hline $\begin{array}{l}\text { Total } \\
\text { Manufacturas }\end{array}$ & 436851 & 100.00 & 4661062 & 100.00 & 367793 & 100.00 & 1480821 & 100.00 & 1586991 & 100.00 \\
\hline Micro & 404156 & 92.52 & 1080713 & 23.19 & & & & 2.85 & 64912 & 4.09 \\
\hline Pequeña & 22349 & 5.12 & 467197 & 10.02 & 24201 & 6.58 & 63571 & 4.29 & 69840 & 4.40 \\
\hline Mediana & 7113 & 1.63 & 797907 & 17.12 & 65407 & 17.78 & 259316 & 17.51 & 279994 & 17.64 \\
\hline Grande & 3233 & 0.74 & 2315245 & 49.67 & 262389 & 71.34 & 1115782 & 75.35 & 1172245 & 73.87 \\
\hline
\end{tabular}

Fuente: elaboración propia con base en los Censos Económicos de 2009. Los datos fueron clasificados por INEGI tomando como referencia los criterios de estratificación empresarial vigentes en 2004 y 2009.

\section{Evolución en el periodo 1999-2009}

Si bien se observa en el cuadro 4 que aumentó el número de empresas micro y grandes, también se detecta que disminuyó el de las pequeñas y medianas; ello es indicio de: a) Una concentración que más adelante se evaluará si es buena o mala en el contexto de la complementariedad que debe existir entre los cuatro tamaños de las empresas manufactureras, es decir, en la relación técnica (producción de bienes inferiores, intermedios y superiores o finales) que debería prevalecer entre ellas para optimizar el uso de su capacidad de planta productiva instalada; b) Una posible escasez del crédito para su operación exitosa. (Sánchez: 2009).

El cuadro 5, sus datos indican que en el país el empleo aumentó 10.1\% y que ello se debió principalmente al incremento porcentual observado en las empresas micro (39.8\%) y en las grandes (13.3\%), en virtud de que disminuyó la capacidad de crear empleo en las pequeñas y medianas. 
Afortunadamente en todos los tamaños de empresas se detectó que las remuneraciones aumentaron en el periodo de análisis; sin embargo, nuevamente fue notorio el mayor dinamismo porcentual en las micro y grandes empresas.

En lo que atañe a la producción generada en este lapso es interesante ver en el cuadro 7 que a nivel nacional se incrementó el valor agregado censal bruto, $154.8 \%$, mismo que fue ocasionado principalmente por las medianas (121.6\%) y grandes empresas (191.6\%). Aquí conviene señalar que en valores absolutos, fueron modestos los incrementos que tuvieron las micro y pequeñas empresas, en tanto que en las medianas y grandes más que se duplicó dicho valor.

El notorio incremento en el valor censal bruto de las medianas y grandes empresas antes descrito, también se explica en mucho por la mayor disponibilidad de planta que tienen, ya que sus activos fijos aumentaron porcentualmente en forma significativa (ver cuadro 8). También es conveniente indicar que en la micro empresas se contrajo la inversión en este rubro y como se vio en el cuadro 6, ello propició la caída en su producción; lo anterior relacionado con su participación porcentual en los indicadores de los cuadros $3.4 \mathrm{y}$ 5 induce a pensar que estas tienen un serio problema de productividad porque en 2009 eran más, aumentaron sus trabajadores y sus remuneraciones correspondientes, pero ahora con la reducción en sus activos fijos producen menos que en 1999.

\section{Cuadro 4}

Evolución del número de empresas manufactureras de 1999 a 2009

\begin{tabular}{|l|r|r|r|}
\hline \multirow{2}{*}{$\begin{array}{c}\text { Tamaño de } \\
\text { Empresa }\end{array}$} & \multicolumn{2}{|c|}{ Número de empresas } & \multicolumn{2}{c|}{ Evolución\% } \\
\cline { 2 - 4 } & $\mathbf{1 9 9 9}$ & $\mathbf{2 0 0 9}$ & $\mathbf{1 9 9 9 - 2 0 0}$ \\
\hline Total nacional & 344118 & 436851 & 26.9 \\
\hline Micro & 310118 & 404156 & 30.3 \\
\hline Pequeñas & 22739 & 22349 & -1.7 \\
\hline Medianas & 8228 & 7113 & -13.6 \\
\hline Grandes & 3033 & 3233 & 6.6 \\
\hline
\end{tabular}

Fuente: elaboración propia con base en los Censos Económicos de 1999 y de 2009. Los datos de 1999 los clasifiqué tomando como referencia los criterios de estratificación empresarial vigentes en 2004 y 2009. 


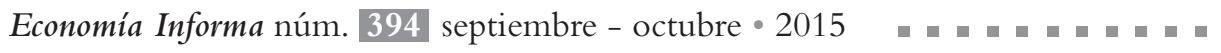

\section{Cuadro 5}

Evolución del personal ocupado por las empresas manufactureras de 1999 a 2009

\begin{tabular}{|l|r|r|r|}
\hline \multirow{2}{*}{$\begin{array}{l}\text { Tamaño de } \\
\text { Empresa }\end{array}$} & \multicolumn{2}{|c|}{ Personal Ocupado } & \multicolumn{1}{c|}{ Evolución\% } \\
\cline { 2 - 4 } & $\mathbf{1 9 9 9}$ & $\mathbf{2 0 0 9}$ & $\mathbf{1 9 9 9 - 2 0 0 9}$ \\
\hline Total nacional & 4232322 & 4661062 & 10.1 \\
\hline Micro & 773288 & 1080713 & 39.8 \\
\hline Pequeñas & 499473 & 467197 & -6.5 \\
\hline Medianas & 916654 & 797907 & -13 \\
\hline Grandes & 2042907 & 2315245 & 13.3 \\
\hline
\end{tabular}

Fuente: elaboración propia con base en los Censos Económicos de 1999 y 2009. Los datos de 1999 los clasifiqué tomando como referencia los criterios de estratificación empresarial vigentes en 2004 y 2009.

\section{Cuadro 6}

Evolución de las remuneraciones obtenidas por el personal ocupado por las empresas manufactureras de 1999 a 2009

\begin{tabular}{|l|r|r|r|}
\hline \multirow{2}{*}{$\begin{array}{c}\text { Tamaño de } \\
\text { Empresa }\end{array}$} & \multicolumn{2}{|c|}{ Millones de pesos } & \multicolumn{1}{c|}{ Evolución \% } \\
\cline { 2 - 4 } & $\mathbf{1 9 9 9}$ & $\mathbf{2 0 0 9}$ & $\mathbf{1 9 9 9 - 2 0 0 9}$ \\
\hline Total nacional & 199043 & 367793 & 84.8 \\
\hline Micro & 7341 & 15797 & 100.2 \\
\hline Pequeñas & 14906 & 24201 & 63.4 \\
\hline Medianas & 43910 & 65407 & 49 \\
\hline Grandes & 132886 & 262389 & 97.5 \\
\hline
\end{tabular}

Fuente: elaboración propia con base en los Censos Económicos de 1999 y 2009. Los datos de 1999 los clasifiqué tomando como referencia los criterios de estratificación empresarial vigentes en 2004 y 2009.

\section{Cuadro 7 \\ Evolución del valor agregado censal bruto obtenido por las empresas manufactureras de 1999 a 2009}

\begin{tabular}{|l|r|r|r|}
\hline \multirow{2}{*}{$\begin{array}{c}\text { Tamaño de } \\
\text { Empresa }\end{array}$} & \multicolumn{2}{|c|}{ Millones de pesos } & \multicolumn{1}{c|}{ Evolución\% } \\
\cline { 2 - 4 } & $\mathbf{1 9 9 9}$ & $\mathbf{2 0 0 9}$ & \multicolumn{1}{|c|}{ 1999-2009 } \\
\hline Total nacional & 581113 & 1480821 & 154.8 \\
\hline Micro & 46173 & 42153 & -8.7 \\
\hline Pequeñas & 35328 & 63571 & 79.9 \\
\hline Medianas & 117020 & 259316 & 121.6 \\
\hline Grandes & 382592 & 1115782 & 191.6 \\
\hline
\end{tabular}

Fuente: elaboración propia con base en los Censos Económicos de 1999 y 2009. Los datos de 1999 los clasifiqué tomando como referencia los criterios de estratificación empresarial vigentes en 2004 y 2009. 


\section{Cuadro 8}

Evolución de los activos fijos con los cuales las empresas manufactureras obtuvieron su valor agregado censal bruto

de 1999 a 2009

\begin{tabular}{|l|r|r|r|}
\hline \multirow{2}{*}{$\begin{array}{c}\text { Tamaño de } \\
\text { Empresa }\end{array}$} & \multicolumn{2}{|c|}{ Millones de pesos } & \multicolumn{1}{c|}{ Evolución\% } \\
\cline { 2 - 4 } & \multicolumn{1}{|c|}{$\mathbf{1 9 9 9}$} & \multicolumn{1}{|c|}{$\mathbf{2 0 0 9}$} & \multicolumn{1}{c|}{$\mathbf{1 9 9 9 - 2 0 0 9}$} \\
\hline Total nacional & 887555 & 1586991 & 78.8 \\
\hline Micro & 89139 & 64912 & -27.2 \\
\hline Pequeñas & 40055 & 69840 & 74.4 \\
\hline Medianas & 172664 & 279994 & 62.2 \\
\hline Grandes & 585698 & 1172245 & 100.1 \\
\hline
\end{tabular}

Fuente: elaboración propia con base en los Censos Económicos de 1999 y 2009. Los datos de 1999 los clasifiqué tomando como referencia los criterios de estratificación empresarial vigentes en 2004 y 2009.

\section{Conclusiones preliminares}

1. La primera hipótesis de trabajo se verifica plenamente porque, en lo que se refiere al uso del crédito para capital de trabajo, el aumento en las remuneraciones al personal ocupado como en la producción generada ponen de manifiesto que las empresas no tienen problemas serios de liquidez, dado que el pago de salarios y la compra de materias primas son los principales conceptos en que se aplican los recursos de capital de trabajo.

2. En lo que atañe a la segunda y tercera: ampliación de la capacidad de la planta productiva, y sustitución de procesos de fabricación, parece que tampoco hubo problemas dado que la información relativa a este rubros revela que aumentaron los activos fijos considerablemente, los cuales para poder utilizarlos, invariablemente son acompañados de sus procesos de fabricación correspondientes, situación que no es indicativa de una urgente necesidad de apoyar con crédito la renovación o ampliación de la planta productiva del sector manufacturero del país.

3. Siendo pocas, las grandes empresas manufactureras generaron la mayor parte de la producción en el periodo de análisis.

4. No son las medianas ni las grandes empresas manufactureras las que pudieran necesitar urgentemente de apoyos crediticios, ya que aumentaron de manera significativa sus inversiones en activos fijos y el valor agregado censal bruto que producen con personal mejor remunerado con respecto a los otros dos estratos empresariales. Estos resultados inducen a investigar 
si ha proliferado la producción de bienes intensivos en el factor capital en detrimento del factor trabajo.

5. De acuerdo a la evolución de sus indicadores de operación técnica, posiblemente son las micro y las pequeñas empresas las que tienen más problemas para su operación productiva y rentable, mismos que convendría conocer con ánimos de detectar si el financiamiento es uno de los principales obstáculos para que sean competitivas y, en caso de que así sea, reconocer la gran importancia para el desarrollo empresarial que le atribuye el Ejecutivo federal en la reforma financiera que instrumentó hace un año y medio.

6. Al respecto, al no contener de manera explícita los Censos Económicos dichos problemas, para verificar la presunción anterior decidí analizar los principales problemas que dicen tener los empresarios que administran los micronegocios en el país, cuya opinión INEGI obtiene periódicamente en la Encuesta Nacional de Micronegocios, Enamin. Al respecto, cabe señalar que INEGI reportó en esta encuesta la opinión de 9.2 millones de empresarios en cinco sectores económicos, en tanto que los resultados definitivos de los Censos Económicos de 2014 prácticamente a menos de la mitad: 4.2 millones en cuatro sectores económicos. Lo anterior permite inferir que la opinión de los primeros es más representativa para el diseño de políticas económicas.

\section{Identificación de los principales problemas que tienen estas empresas}

Considero que ellos constituyen la referencia básica para determinar si la falta de crédito es el principal obstáculo para el desarrollo empresarial y por consiguiente, el fundamento para la elaboración de la política pública que haga de este sector la bujía del sector económico. Así, el cuadro 9 muestra que la Enamin 2013, con sus resultados confirma que en opinión de los empresarios la falta de crédito no es el principal problema de la inmensa mayoría de las empresas en México, puesto que sólo el 2.76\% dijo que lo era.

Lo anterior lo corroboran los resultados definitivos de los Censos Económicos 2014 ya que INEGI informa que el 65.5\% de los micro empresarios no se financian con el crédito bancario sino en las cajas de ahorro, con familiares, proveedores y prestamistas privados. 


\section{Cuadro 9}

Opinión de los empresarios sobre sus principales problemas en 2013

\begin{tabular}{|c|c|c|c|c|c|c|}
\hline \multirow{2}{*}{ Indicador } & \multicolumn{3}{|c|}{ Nacional } & \multicolumn{3}{|c|}{ Porcentaje } \\
\hline & Total & Hombres & Mujeres & Total & Hombres & Mujeres \\
\hline $\begin{array}{l}\text { Principal problemática } \\
\text { presentada }\end{array}$ & 9192490 & 4439169 & 4753321 & 100 & 100 & 100 \\
\hline 1. Bajaron las ventas & 2248828 & 1021179 & 1227649 & 24.46 & 23.00 & 25.83 \\
\hline $\begin{array}{l}\text { 2. Falta de crédito o } \\
\text { financiamiento }\end{array}$ & 253352 & 123256 & 130096 & 2.76 & 2.78 & 2.74 \\
\hline $\begin{array}{l}\text { 3. Aumento de los precios } \\
\text { en insumos y/o mercancías }\end{array}$ & 799172 & 375929 & 423243 & 8.69 & 8.47 & 8.90 \\
\hline $\begin{array}{l}\text { 4. El negocio es menos } \\
\text { rentable }\end{array}$ & 133267 & 61627 & 71640 & 1.45 & 1.39 & 1.51 \\
\hline 5. Competencia excesiva & 1601699 & 904555 & 697144 & 17.42 & 20.38 & 14.677 \\
\hline 6. Problemas con los clientes & 345400 & 122172 & 223228 & 3.76 & 2.75 & 4.70 \\
\hline 7. Otros & 1210141 & 729594 & 480547 & 13.16 & 16.44 & 10.11 \\
\hline 8. Sin problemas & 2600631 & 1100857 & 1499774 & 28.29 & 24.80 & 31.55 \\
\hline
\end{tabular}

Fuente: Encuesta Nacional de Micronegocios de INEGI 2013.

Resumen: Derivada del análisis de la información empresarial comprendida de los cuadros 1 al 8 , se infiere que la política pública instrumentada para la promoción del desarrollo económico, se ha formulado sin considerar la opinión de la mayoría de los empresarios. Todo apunta al hecho de que ahora con esta Iniciativa el Estado mexicano pretende resolver los problemas operacionales de las empresas, en particular la de los micronegocios. Ni por asomo prevé solucionar sus principales problemas estructurales que se describen en el cuadro 9.

\section{Consecuencias}

1. Los resultados de los Censos Económicos de 2014 (que ya comprenden los primeros efectos de la reforma financiera) dados a conocer al público por INEGI en diciembre pasado permiten inferir que el crédito no promovió el desarrollo empresarial, en virtud de que en opinión de esta institución de 2009 a 2014 el universo de empresas se contrajo en 300 mil negocios (Periódico La Razón: 2014) y que México es un país de micronegocios vulnerables (Sojo, íbid).

2. Derivado de lo anterior el mismo funcionario informó que en el lustro de 2009 a 2014 "se crearon 520 mil 459 empresas, un avance de anual de 1.95 por ciento en ese lapso, tasa inferior al crecimiento anual registrado en los 
cinco años previos, que fueron en promedio de 3.7 por ciento, mediante la creación de 853 mil 948 empresas".

\section{Conclusiones y recomendaciones}

\section{Conclusiones}

1. Falló la reforma financiera en el uso del crédito para fomentar el desarrollo empresarial, en virtud der que el número de empresas disminuyó de 2009 a 2014.

2. Vía el crédito a las empresas manufactureras el Estado mexicano no generó suficientes empleos bien remunerados y permanentes.

3. Según los resultados de operación de las empresas manufactureras, para sus empresarios el crédito no es su principal problema, como tampoco para los empresarios de los micronegocios.

4. Carece de evidencia estadística el efecto del crédito visualizado en la reforma financiera como elemento significativo para resolver los problemas estructurales del sector empresarial, base del desarrollo económico nacional.

5. Causa extrañeza el énfasis que le pone a su promoción el gobierno en la reforma financiera.

6. Ahora como antaño, según los empresarios de la industria manufacturera como los de otros sectores económicos, la banca comercial y la de desarrollo no son su principal fuente de financiamiento, como tampoco el gobierno es el principal demandante de sus bienes manufacturados.

7. Preocupa que la inmensa mayoría de los agentes económicos, los que producen; no sean sujetos de crédito. Es desconcertante ver que en plena época de contracción de los ingresos fiscales, la banca comercial promueva con ahínco el crédito para el consumo y que el gobierno sea indiferente.

8. Derivado de lo anterior, ¿Por qué una persona como consumidor es mejor sujeto de crédito que como productor? ¿qué no tiene más garantías para la recuperación del crédito dicha persona como fabricante que como consumidor ya que cuenta con activos líquidos y fijos para pagar sus pasivos contraídos?

9. En este contexto, cabe preguntar: ¿Ha fallado, es insuficiente, el programa oficial de garantías de la banca de desarrollo para asegurar ante la banca comercial la recuperación de los crédito? ¿Qué explicación da la SHCP?

10. Ante la caída de los ingresos provenientes del petróleo, ¿Sigue siendo inteligente la promoción indiscriminada que hace el gobierno y los bancos 
comerciales del crédito? ¿No sería mejor priorizar su aplicación en actividades prioritarias y estratégicas con alto contenido nacional en sus procesos de fabricación, de insumos, materias primas y mano de obra local que aumentan la capacidad ociosa de la planta productiva del país?

11. Independientemente de la disponibilidad de recursos oficiales y privados para el fomento económico, así como de que si una persona es o no sujeto de crédito y del destino que se le asigne al crédito, ¿No sería mejor que la reforma financiera incidiera en la baja del costo del dinero? ¿Por qué la asimetría entre las tasas de interés pasivas y activas de la banca comercial? ¿Cómo es que a las personas como ahorradores les paga 3\% anual y como usuarios del crédito hasta $40 \%$ ?

12. Lo anterior quizás explique en parte, pero de manera contundente, el por qué, según INEGI, disminuyó el número de empresas de 2009 a 2014.

\section{Recomendaciones}

1. El gobierno federal debe escuchar, conversar y consensar con los empresarios sus políticas públicas de aliento a la economía nacional.

2. Ante la escasez de recursos públicos, la reforma financiera debe revisarse para fomentar el crédito de manera selectiva y no indiscriminadamente, asignándolo preferentemente a los fabricantes con respecto a los consumidores, ya que la depreciación del peso está encareciendo las importaciones y ello brinda la oportunidad de fortalecer el modelo económico de desarrollo endógeno impulsando la producción de bienes manufacturados con mano de obra y materias primas locales. Situación que en turno genera el circulo virtuoso puesto que al haber más ingresos gracias al efecto multiplicador y al incremento en el uso de la capacidad de planta productiva del país, aumentará la demanda efectiva, la recaudación fiscal, el empleo permanente y mejor remunerado, es decir, el bienestar de la población.

3. La reforma financiera, simultáneamente con el crédito, debe impulsar una mayor competencia entre los bancos comerciales para que éstos reduzcan las comisiones que cobran por sus servicios, al igual que para reducir la enorme diferencia que prevalece entre sus tasas de interés activas y pasivas.

4. En este contexto de impulso al desarrollo empresarial, la reforma financiera debe exigir que los bancos de segundo piso incrementen sus programas de garantías, para que haya un mayor número de sujetos de crédito empresariales. 
5. La reforma financiera debe favorecer en la banca de desarrollo su ampliación como banca de primer piso de sus operaciones crediticias, de asistencia técnica y de impulso a programas prioritarios como los ecológicos, los de desarrollo empresarial, etc, para que establezca una relación directa con los empresarios y para que su derrama crediticia en particular, no dependa de los movimientos de tesorería de los bancos comerciales.

6. La reforma financiera debe revisarse periódicamente para evaluar los avances y retrocesos en congruencia con el resto de las reformas estructurales en vigor y cuyo sustento es el Plan Nacional de Desarrollo 2013-2018.

7. La reforma financiera, para bajar el costo del dinero, debe hacer que los agentes financieros del gobierno federal que buscan anualmente recursos en los mercados financieros internacionales para la contratación de la deuda que autoriza el H. Congreso de la Unión, éstos también se diversifiquen hacia otras fuentes de financiamiento diferentes a las del FMI, Banco Mundial BID, etc. por ejemplo, hacia el banco que surgió cuando entró en vigor el TLC, de los países emergentes: China., Luxemburgo, UE.

8. La reforma financiera debe inducir en el sistema bancario la aplicación de recursos a fondo perdido en regiones deprimidas, con escaso desarrollo económico pero con abundancia de mano de obra y recursos naturales susceptibles de aprovechamiento en el mediano y largo plazo.

9. Se sugiere que dentro de su política pública de fomento empresarial, el gobierno le asigne mayor prioridad a la solución de los problemas estructurales de las empresas. Concretamente, se recomienda que anualmente, con base en los resultados de operación de las unidades de producción y de las opiniones de los empresarios, el Ejecutiva Federal proponga al H. Congreso de la Unión la política pública de financiamiento para el desarrollo económico fincado en el fomento preferencial de la competitividad de las Mipymes.

10. Como un serio ejercicio de planeación, de programación y de ejecución, se propone que para el mejor aprovechamiento de la planta productiva y para la generación de empleos permanentes y bien remunerados, el gobierno y los empresarios visualicen el eslabonamiento técnico de los cuatro tamaños de empresas en las cadenas de producción: la fabricación de bienes inferiores, intermedios y bienes finales. 


\section{Bibliografía}

Diario Oficial de la Federación, Internet, 2015.

INEGI, "Censos Económicos de 1999, 2004, 2009 y 2014";

, “Encuesta Nacional de Micronegocios," STPS, INEgI, 1998, 2002, 2008, 2010 y 2013.

Periódico La Razón, Negocios, 11 de diciembre de 2014, página 24, México.

Sánchez, Barajas Genaro, (2007), Perspectiva de las micro y pequeñas empresas como factores del desarrollo económico de México. México: Centro de Estudios para el Desarrollo Nacional.

(2009), Relocalización de las empresas industriales, de 1999 a 2004, escuela Superior de Economía, IPN, Revista No. 21, enero-marzo, 2009.

(2012) Atractividad para la inversión de las 32 entidades federativas de México, Curso en el posgrado de la Universidad de Economía de Praga, República Checa, en noviembre de 2012.

SHCP," Iniciativa de Reforma Financiera", Presidencia de la República, 8 de mayo de 2013, así como la exigencia del Presidente de la República a la banca de desarrollo de que "aumenten el crédito en 10\% con respecto a 2012", escuchada por el suscrito en TV durante la primera quincena del mes de junio de 2013. 